\title{
Generalising the simultaneous computation of the DFTs of two real sequences using a single $N$-point DFT
}

\author{
Vitor Silva*, Fernando Perdigão \\ Institute of Telecommunications, Department of Electrical and Computer Engineering, Pole II of University Coimbra, \\ Coimbra 3030-290, Portugal
}

\begin{abstract}
A general approach to the problem of simultaneous computation of the discrete Fourier transform (DFT) of two sequences of length $N$, which may be real, imaginary, conjugated symmetric or conjugated anti-symmetric, using a single $N$-point DFT of a complex sequence is presented. The framework developed is applied to the simultaneous computation of two DFTs, one DFT and one inverse DFT (IDFT) or two IDFTs for real $N$-point sequences. (C) 2002 Published by Elsevier Science B.V.
\end{abstract}

Keywords: DFT; IDFT; DMT

\section{Introduction}

Efficient DFT computation has been a challenge to the signal processing community over the last several decades [3,5]. In this communication, we will introduce and generalise the problem of simultaneous computation of the DFTs of two $N$ free parameter sequences using a single $N$-point DFT of a $2 N$ parameter complex sequence. Each input sequence has length $N$ and is either real, imaginary, complex symmetric or complex anti-symmetric, in order to be defined with only $N$ free parameters.

Related work on this subject has been presented recently [2,4], but a more generic and systematic approach to this problem can be developed as will be shown in the following sections. In particular, we illustrate this approach with the practical cases of computation of either two DFTs, one DFT and one IDFT

\footnotetext{
* Corresponding author.

E-mail addresses: vitor@co.it.pt (V. Silva), fp@co.it.pt (F. Perdigão).
}

or two IDFTs of $N$ free parameter sequences with a single $N$-point DFT. These computation tools are important, for example, for the development of modern discrete multicarrier modulation (DMT) modems [1].

\section{Definitions and properties}

In order to simplify the notation, we assume that a sequence $z[n]$ of finite length $N$ and its DFT, $Z(k)$, are both periodic sequences of period $N$, so we can write $z[-n]$ instead of $z[N-n]$ or $z[-n \bmod N]$. A generic complex sequence $z[n]$ can always be expressed as a sum of its real and imaginary parts or as a sum of its conjugate symmetric (CS) part and conjugate anti-symmetric (CA) part,

$z[n]=z_{\mathrm{R}}[n]+j z_{\mathrm{I}}[n]=z_{\mathrm{cs}}[n]+z_{\mathrm{ca}}[n]$,

where $z_{\mathrm{R}}[n]=\operatorname{Re}\{z[n]\}$ and $z_{\mathrm{I}}[n]=\operatorname{Im}\{z[n]\}$ and the CS and CA parts of $z[n]$ are complex sequences [3,5], given by

$$
\begin{aligned}
& z_{\mathrm{cs}}[n]=\frac{1}{2}\left(z[n]+z^{*}[-n]\right)=z_{\mathrm{cs}}^{*}[-n], \\
& z_{\mathrm{ca}}[n]=\frac{1}{2}\left(z[n]-z^{*}[-n]\right)=-z_{\mathrm{ca}}^{*}[-n] .
\end{aligned}
$$


If $z[n]$ is real the CS and CA parts are designated by the even and the odd parts, respectively, [3,5]. Furthermore, the real and imaginary parts could be decomposed into its even and odd parts, and the CS and CA parts into real and imaginary parts, resulting in,

$$
\begin{aligned}
z[n] & =\left(z_{\mathrm{R}_{\mathrm{e}}}[n]+z_{\mathrm{R}_{\mathrm{o}}}[n]\right)+j\left(z_{\mathrm{I}_{\mathrm{e}}}[n]+z_{\mathrm{I}_{\mathrm{o}}}[n]\right) \\
& =\left(z_{\mathrm{cs}_{\mathrm{R}}}[n]+j z_{\mathrm{cs}_{\mathrm{I}}}[n]\right)+\left(z_{\mathrm{ca}_{\mathrm{R}}}[n]+j z_{\mathrm{ca}_{\mathrm{I}}}[n]\right),
\end{aligned}
$$

where $z_{\mathrm{R}_{\mathrm{e}}}[n]=z_{\mathrm{cSR}_{\mathrm{R}}}[n], z_{\mathrm{R}_{\mathrm{o}}}[n]=z_{\mathrm{ca}_{\mathrm{R}}}[n], z_{\mathrm{I}_{\mathrm{e}}}[n]=z_{\mathrm{cal}_{\mathrm{I}}}[n]$ and $z_{\mathrm{I}_{\mathrm{o}}}[n]=z_{\mathrm{CS}_{\mathrm{I}}}[n]$, due to the symmetry properties.

The DFT exhibits duality between real and CS and between imaginary and CA sequences. Thus, the DFT of a sum of a real and an imaginary sequence has in its CS and CA parts the two original DFTs. Similarly, the DFT of a sum of a CS with a CA sequence has in its real and imaginary parts the two original DFTs.

Any of the real, imaginary, CS or CA sequences of length $N$ have only $N$ free parameters in opposition to a generic complex sequence which have $2 N$ parameters. Forming conveniently a complex sequence of $2 N$ free parameters, $z[n]$, with the full information of two sequences with $N$ free parameters, it is always possible to recover the partial DFTs from the DFT of $z[n], Z(k)$. As suggested by (4), the only restriction is that the real and imaginary parts of $z[n]$ must contain one even and one odd sequence, in order to have the corresponding components in $Z(k)$ separable.

\section{Problem formulation}

Given two real sequences of length $N, a[n]$ and $b[n]$, which we decompose into its even and odd parts, $a_{\mathrm{e}}[n], a_{\mathrm{o}}[n], b_{\mathrm{e}}[n]$ and $b_{\mathrm{o}}[n]$, we can form two sequences (with $N$ free parameters) as

$x[n]=c_{1} a_{\mathrm{e}}[n]+c_{2} a_{\mathrm{o}}[n]$,

$y[n]=c_{3} b_{\mathrm{e}}[n]+c_{4} b_{\mathrm{o}}[n]$,

where $c_{1}, c_{2}, c_{3}, c_{4} \in \mathbb{C}$. If the constants are real and/or imaginary the sequences would be real, imaginary, CS or CA. For example, if $c_{1}$ is real and $c_{2}$ is imaginary, then $x[n]$ is CS. From the DFT properties [5] result

$$
\begin{aligned}
& X(k)=c_{1} A_{\mathrm{R}}(k)+j c_{2} A_{\mathrm{I}}(k), \\
& Y(k)=c_{3} B_{\mathrm{R}}(k)+j c_{4} B_{\mathrm{I}}(k),
\end{aligned}
$$

where $A_{\mathrm{R}}(k)$ and $B_{\mathrm{R}}(k)$ are even and $A_{\mathrm{I}}(k)$ and $B_{\mathrm{I}}(k)$ are odd sequences. If we have two sequences, $x[n]$ and $y[n]$, conforming to (5) and (6), first we identify the real sequences $a[n]$ and $b[n]$ and then form the sequence $z_{1}[n]$ or $z_{2}[n]$ according to

$$
\begin{aligned}
z_{1}[n] & =a_{\mathrm{e}}[n]+a_{\mathrm{o}}[n]+j\left(b_{\mathrm{e}}[n]+b_{\mathrm{o}}[n]\right) \\
& =a[n]+j b[n], \\
z_{2}[n] & =a_{\mathrm{e}}[n]+b_{\mathrm{o}}[n]+j\left(a_{\mathrm{o}}[n]+b_{\mathrm{e}}[n]\right) .
\end{aligned}
$$

Since both real and imaginary parts of $z_{1}[n]$ and $z_{2}[n]$ contain an even and an odd sequence, the respective DFT also has in its real and imaginary parts an even and an odd DFT component. From (9) and (10) we obtain

$$
\begin{aligned}
& Z_{1}(k)=A_{\mathrm{R}}(k)+j A_{\mathrm{I}}(k)+j B_{\mathrm{R}}(k)-B_{\mathrm{I}}(k), \\
& Z_{2}(k)=A_{\mathrm{R}}(k)+j B_{\mathrm{I}}(k)-A_{\mathrm{I}}(k)+j B_{\mathrm{R}}(k) .
\end{aligned}
$$

The four DFT components are obtained from $Z_{1}(k)$ or $Z_{2}(k)$ as $A_{\mathrm{R}}(k)=Z_{1_{\mathrm{R}_{\mathrm{e}}}}(k)=Z_{2_{\mathrm{R}_{\mathrm{e}}}}(k), A_{\mathrm{I}}(k)=$ $Z_{1_{\mathrm{I}_{\mathrm{o}}}}(k)=-Z_{2_{\mathrm{R}_{\mathrm{o}}}}(k), B_{\mathrm{R}}(k)=Z_{1_{\mathrm{I}_{\mathrm{e}}}}(k)=Z_{2_{\mathrm{I}_{\mathrm{e}}}}(k)$ and $B_{\mathrm{I}}(k)=-Z_{\mathrm{R}_{\mathrm{R}_{\mathrm{o}}}}(k)=Z_{\mathrm{I}_{\mathrm{I}}}(k)$.

Then, according to (7) and (8), $X(k)$ and $Y(k)$ are obtained from $Z_{1}(k)$ or $Z_{2}(k)$ through the following equations:

$$
\begin{aligned}
X(k) & =c_{1} Z_{1_{\mathrm{R}_{\mathrm{e}}}}(k)+j c_{2} Z_{\mathrm{I}_{\mathrm{I}}}(k) \\
& =c_{1} Z_{2_{\mathrm{R}_{\mathrm{e}}}}(k)-j c_{2} Z_{2_{\mathrm{R}_{\mathrm{o}}}}(k), \\
Y(k) & =c_{3} Z_{1_{\mathrm{I}_{\mathrm{e}}}}(k)-j c_{4} Z_{1_{\mathrm{R}_{\mathrm{o}}}}(k) \\
& =c_{3} Z_{2_{\mathrm{I}_{\mathrm{e}}}}(k)+j c_{4} Z_{2_{\mathrm{I}_{\mathrm{o}}}}(k) .
\end{aligned}
$$

Obviously, there are other two forms to obtain similar results [2], but they amount to an exchange of the roles of $a[n]$ and $b[n]$ in (9) and (10). In the following sections we apply these expressions to the cases of simultaneous computation of two DFTs, one DFT and one IDFT or two IDFTs for real sequences.

\section{DFTs of two real sequences}

If $c_{1}=c_{2}=c_{3}=c_{4}=1$ in (5) and (6) we have the case of two real sequences: $x[n]=a[n]$ and $y[n]=b[n]$. Then $z_{1}[n]=x[n]+j y[n]$, which corresponds to the usual approach [3], of taking the DFTs of two real sequences with the computation of only one $N$-point DFT. The result in compact form is $X(k)=Z_{1_{\mathrm{cs}}}(k)$ and $j Y(k)=Z_{1_{\mathrm{ca}}}(k)$. In terms of $Z_{2}(k)$ the result is $X(k)=Z_{2_{\mathrm{R}_{\mathrm{e}}}}(k)-j Z_{2_{\mathrm{R}_{\mathrm{o}}}}(k)$ and $Y(k)=Z_{2_{\mathrm{I}_{\mathrm{e}}}}(k)+j Z_{2_{\mathrm{I}_{\mathrm{o}}}}(k)$. 


\section{DFT of a real sequence and IDFT of a CS sequence}

The problem of the simultaneous computation of one DFT and one IDFT for length $N$ real sequences has been recently reported and solved using some methods developed in an ad hoc way $[2,4]$. A more systematic approach comes from (5) and (6) when associated to a real and to a CS sequence.

An IDFT can be viewed as a DFT, i.e., $x[n]=$ $\operatorname{DFT}\{X(-k) / N\}$, or, assuming $x[n]$ periodic, DFT $\{X(k)\}=N x[-n]$. Beginning with a CS sequence, $W(k)$, and a real sequence $y[n]$, we want to obtain $w[n]$ (real) and $Y(k)$. One possibility is to make $c_{3}=c_{4}=1(y[n]=b[n]$ in $(6))$ and

$x[n]=\frac{1}{N} W(-n)=\frac{1}{N}\left(W_{\mathrm{R}}(n)-j W_{\mathrm{I}}(n)\right)$,

so that $X(k)=w[k]$. In this case, we could choose the following assignments:

$a_{\mathrm{e}}[n]=\frac{1}{N} W_{\mathrm{R}}(n), \quad a_{\mathrm{o}}[n]=-\frac{1}{N} W_{\mathrm{I}}(n)$,

which means $c_{1}=1$ and $c_{2}=j$. Using (13) and (14) the result is

$w[n]=Z_{1_{\mathrm{R}_{\mathrm{e}}}}(n)-Z_{1_{\mathrm{I}_{\mathrm{o}}}}(n)=Z_{2_{\mathrm{R}}}(n)$,

$Y(k)=-j Z_{1_{\mathrm{ca}}}(k)=Z_{2_{\mathrm{I}_{\mathrm{e}}}}(k)+j Z_{2_{\mathrm{I}_{\mathrm{o}}}}(k)$.

All methods reported in $[2,4]$ are particular cases of the approach outlined.

\section{Two IDFTs for real sequences}

Another important application is the simultaneous computation of two IDFTs that lead to real sequences. Suppose we have $W(k)$ and $V(k)$, both CS, and wish to obtain $w[n]$ and $v[n]$, both real. In this case we take, as in (15), $x[n]=W(-n) / N$ and $y[n]=V(-n) / N$.
For $a[n]$ we choose (16) and, similarly, for $b[n]$ we choose

$b_{\mathrm{e}}[n]=\frac{1}{N} V_{\mathrm{R}}(n), \quad b_{\mathrm{o}}[n]=\frac{1}{N} V_{\mathrm{I}}(n)$,

which means $c_{1}=c_{3}=1, c_{2}=j$ and $c_{4}=-j$. The required sequences are then given by (17) and, from (14), by

$v[n]=Y(n)=Z_{1_{\mathrm{I}_{\mathrm{e}}}}(n)-Z_{1_{\mathrm{R}_{\mathrm{o}}}}(n)=Z_{\mathrm{2}_{\mathrm{I}}}(n)$.

Notice that if we form the sequence $z_{2}[n]$ with (16) and (19) then $w[n]$ and $v[n]$ are simply the real and imaginary parts of $Z_{2}(n)$, respectively.

\section{Conclusions}

A general approach to the problem of simultaneous computation of the DFTs of two length $N$ real or complex symmetric sequences using a single $N$-point DFT has been presented. We have shown how to solve generically the problem of computing simultaneously either two DFTs, one DFT and one IDFT or two IDFTs for real sequences, with a single $N$-point DFT.

\section{References}

[1] J.A.C. Bingham, ADSL, VDSL and Multicarrier Modulation, Wiley, New York, 2000.

[2] S.S. Kidambi, Simultaneous computation of the DFT of an $N$-point real sequence and the IDFT of an $N$-point complex sequence with conjugate symmetry with a single $N$-point DFT, Signal Processing 81 (2001) 1109-1112.

[3] S.K. Mitra, Digital Signal Processing: a Computer-Based Approach, McGraw-Hill, New York, 1998.

[4] Shay Moshe, David Hertz, On computing DFT of real $N$-point vector and IDFT of DFT-transformed real $N$-point vector via single DFT, IEEE Signal Process. Lett. 6 (1999) 141.

[5] A.V. Oppenheim, et al., Discrete-Time Signal Processing, Prentice-Hall, Englewood Cliffs, NJ, 1989. 